\title{
Efecto de una intervención dietoterapéutica con diferentes aportes de calcio dietario sobre el estado nutricional en estudiantes universitarias con sobrepeso
}

\author{
Effect of an intervention with different contributions of calcium on \\ nutritional status in overweight university students
}

\begin{abstract}
RESUMEN.
Una baja ingesta de calcio se ha asociado con mayor prevalencia de sobrepeso y obesidad. El objetivo fue evaluar el efecto de una intervención dietoterapéutica con diferentes aportes de calcio dietario sobre el estado nutricional, en estudiantes universitarias con sobrepeso. El diseño fue cuasi-experimental $(n=18)$ en dos grupos intervenidos durante 8 semanas. El grupo $1(n=10)$ restringió su consumo de lácteos a $<3$ porciones, mientras que el grupo 2 $(n=8)$ consumieron 5 porciones diarias (leche fluida UHT descremada). La intervención consideró, para ambos grupos, una restricción de $500 \mathrm{kcal} /$ día respecto de su gasto energético total. Se evaluó el estado nutricional por IMC $\left(\mathrm{kg} / \mathrm{m}^{2}\right)$, circunferencia de cintura (CC) y porcentaje de masa grasa (\%MG). Como resultados se obtuvo que, la ingesta de calcio dietario, aumentó en 916,4 mg/día en el grupo de "5 porciones de lácteos", pero disminuyó en -28,9 mg/día en el grupo de "3 porciones de lácteos". Al comparar los grupos, ambos redujeron significativamente $(p<0.05)$ su peso corporal (-3,6 vs. -3,4 kg), IMC (-1,45 vs. $\left.-1,31 \mathrm{~kg} / \mathrm{m}^{2}\right)$, CC $(-4,5 \mathrm{vs} .-6,2 \mathrm{~cm})$ y $\%$ MG (-2,5 vs. $-2,7$ $\%)$. Estos resultados sugieren que aumentar el consumo de lácteos a 5 porciones diarias no presenta beneficios adicionales para la mejora del estado nutricional después de 8 semanas de intervención.
\end{abstract}

Palabras clave: Calcio dietario; Dietoterapia; Pérdida de peso; Sobrepeso.

\footnotetext{
ABSTRACT

A low intake of calcium has been associated with greater risk of overweight and obesity. This work aimed to evaluate the effect of different levels of calcium intake on the nutritional status of overweight female university students. This 8-week intervention included two groups who restricted their calcium intake to $<3$ portions or 5 portions of dairy products per day (nonfat ultra-high temperature processed milk). Height, weight, body mass index (BMI), waist circumference (WC) and body fat percentage (BF) were measured at baseline and post-intervention. The intervention considered for both groups a $500 \mathrm{kcal} /$ day restriction based on their total energy expenditure. The main result
}

Manola Olea ${ }^{1 *}$, Carlos Celis-Morales ${ }^{2,3}$, Gislaine Granfeldt ${ }^{1}$.

\begin{abstract}
*Dirigir correspondencia a: Manola Olea Vidal. Departamento de Nutrición y Dietética, Facultad de Farmacia, Universidad de Concepción, Concepción, Chile. Código postal: 4030000. Celular: +56930535866 . E-mail: manolea@udec.cl
\end{abstract}

of this study showed that dietary calcium intake increased to $916.4 \mathrm{mg} /$ day in the group consuming 5 portions per day but decreased (-28.9 $\mathrm{mg} /$ day) in the group consuming 3 daily portions. Both the 3 and 5 portion intake groups significantly reduced their body weight (-3.6 vs. $-3.4 \mathrm{~kg})$, $B M I\left(-1.45 \mathrm{vs} .-1.31 \mathrm{~kg} / \mathrm{m}^{2}\right), W C(-4.5 \mathrm{vs} .-6.2 \mathrm{~cm})$ and $B F$ $(-2.5$ vs. $-2.7 \%)$. These findings suggest that 5 dairy portions per day compared to 3 does not have additional benefits for improved nutritional status.

Keywords: Calcium; overweight; Diet therapy; Weight loss.

\section{INTRODUCCIÓN}

La prevalencia de sobrepeso y obesidad han mostrado una tendencia creciente a nivel mundial en las últimas 
décadas. En Chile, las cifras alcanzan un 39,8\% y 31,2\% en adultos según Encuesta Nacional de Salud 2017', posicionándolo en los primeros lugares de los países con mayor obesidad en Latino América, después de México. Esta realidad también se observa en estudiantes universitarios, un estudio realizado en universitarios de 11 regiones de Chile, reportó que un $19,8 \%$ de mujeres y un $27,9 \%$ de hombres presentaron sobrepeso y un $4,1 \%$ y un $5,5 \%$ presentaron obesidad respectivamente ${ }^{2}$.

Uno de los factores que se asocia a este alto nivel obesidad en población universitaria es el actual patrón alimentario de los estudiantes. Este se caracteriza por un consumo de alimentos procesados de alta densidad energética y poco variado, lo que ha contribuido al actual perfil epidemiológico de la obesidad y enfermedades crónicas de Chile 3 . La población universitaria no está ajena a esta realidad, desde el punto de vista dietético, se caracteriza por la selección de alimentos poco saludables $4,5,6,18$. Ratner y colaboradores, reportaron una alta frecuencia de consumo de alimentos como pasteles, galletas y dulces, bebidas carbonatadas y frituras, lo cual se asocia con mayor riesgo de obesidad, hipertensión arterial e hipercolesterolemia, y baja frecuencia de consumo diario de frutas, verduras y lácteos, lo que se relaciona con menor consumo de antioxidantes, fibra y calcio ${ }^{2}$. Por otra parte, existen diversos estudios que señalan que los estudiantes, durante el periodo universitario, pueden adquirir hábitos alimentarios inadecuados, los cuales influyen en la aparición del estado nutricional de sobrepeso y obesidad, pudiendo incrementar el riesgo de desarrollar enfermedades crónicas no transmisibles (ECNT's) en la vida adulta ${ }^{2,4}$.

La intervención dietoterapéutica podría contribuir a combatir esa creciente prevalencia de obesidad, sin embargo, sabemos que la efectividad de una intervención dietoterapéutica está determinada por la planificación de un plan alimentario estructurado, equilibrado y variado, con el objetivo de ajustar la ingesta energética a la Tasa Metabólica en Reposo (TMR) del individuo, y rectificar o modificar las alteraciones en el patrón alimentario, si existiesen ${ }^{7}$, distribuyendo los sustratos energéticos de la dieta de manera proporcional, según lo establece la OMS, considerando el aporte de calcio dietario, micronutriente cuyo aporte es insuficiente en la dieta de la población.

Las guías alimentarias para la población chilena, indican un consumo de 3 porciones de lácteos diarios, lo cual equivale a un aporte de $720 \mathrm{mg}$ de calcio/día, lo que no alcanza a cubrir los requerimientos diarios de calcio en una mujer adulta, cuyas recomendaciones diarias de ingesta son de $1.000 \mathrm{mg}$ de calcio/día ${ }^{2,3,8,9}$. Según la Encuesta Nacional de Consumo Alimentario (ENCA), realizada en el año 2015, se observa un consumo promedio de lácteos de $330 \mathrm{ml} /$ día, aportando $360 \mathrm{mg}$ de calcio/día ${ }^{10}$. Esta ingesta en la población chilena podría estar asociada a un mayor riesgo de obesidad y ECNT's.

Investigaciones recientes han reportado que una baja ingesta de calcio se asocia con una mayor prevalencia de obesidad, reportando una relación inversa entre la ingesta de calcio y la masa grasa corporal, favoreciendo su redistribución alejada del abdomen ${ }^{11,12,16}$. El posible efecto anti obesidad del calcio se centra en los eventos postprandiales que pudiesen contribuir, como son el aumento de la termogénesis inducida por la dieta, aumento en oxidación de la grasa corporal, aumento de la lipólisis y disminución en la absorción de grasas, lo cual produce una mayor pérdida de grasa y energía fecal, disminuye la sensación de hambre y por ende disminuye la ingesta de alimentos ${ }^{11}$. Por ende, una de las hipótesis plantea que un aumento de la ingesta de calcio a través de la dieta (lácteos), que no supere la ingesta máxima tolerable de calcio de $2.500 \mathrm{mg}$ de calcio/día $\mathrm{a}^{17}$, podría tener efectos sobre el estado nutricional durante una restricción calórica ${ }^{11,12,15,16}$. Tomando estos antecedentes en cuenta, el objetivo de este trabajo fue evaluar el efecto de una intervención dietoterapéutica con diferentes aportes de calcio dietario sobre el estado nutricional, en estudiantes universitarias con sobrepeso.

\section{MATERIAL Y MÉTODOS Diseño del estudio}

Este estudio de tipo cuasi-experimental incluyó a 18 mujeres con un IMC $\geq 25,0 \mathrm{~kg} / \mathrm{m}^{2}$, las cuales fueron asignadas a dos grupos de intervención. El grupo 1 o también llamado "3 porciones de lácteos" incluyó a 10 mujeres mientras que el grupo 2 llamado " 5 porciones de lácteos" incluyó a 8 mujeres.

La intervención tuvo una duración de 8 semanas y para la cual se realizaron evaluaciones pre y post intervención. Las mujeres fueron reclutadas mediante muestreo por conveniencia. La asignación a los grupos de intervención se realizó de forma aleatoria. En relación a la muestra, esta incluyó estudiantes universitarias de género femenino, con estado de nutricional de sobrepeso, pertenecientes a la carrera de Nutrición y Dietética de la Universidad de Concepción. Los criterios de inclusión fueron: ser mujer, participar en forma voluntaria, edad entre 19-28 años con sobrepeso según IMC $\left(\geq 25,0 \mathrm{~kg} / \mathrm{m}^{2}\right)$ y firmar consentimiento informado. Los criterios de exclusión fueron: estar en periodo de gestación, amamantamiento, tener patología asociada como enfermedad tiroidea y/o anemia, estar en tratamiento con algún sedante, analgésico y/o beta-bloqueadores o no cumplir con los criterios de inclusión.

\section{Diseño de la intervención}

A cada grupo se le realizó una intervención dietoterapéutica, que se inició con un diagnóstico dietético basal individual a través del Registro Dietético Diario de 7 días consecutivos, donde debían registrar su alimentación diaria con detalles en el horario de ingesta de alimentos, tiempos de comidas, minuta y porciones de alimentos en medidas caseras. Para ello, se citó a las participantes a una reunión grupal, donde se les entregaron las indicaciones correspondientes para realizar el Registro Dietético Diario. El formato de registro se envió vía correo electrónico y luego lo reportaron por este mismo medio. Un nutricionista determinó el aporte de energía, de macronutrientes 
y de calcio de los registros, a través del software "Sistema de Evaluación Nutricional" de la Facultad de Farmacia de la Universidad de Concepción, el cual está diseñado en base a la Tabla de Composición Química de los Alimentos Chilenos 2000, que permite realizar un análisis cuantitativo de la dieta.

La atención dietoterapéutica se otorgó a través de tres consultas nutricionales (ingreso y dos controles) y consistió en prescribir el régimen de alimentación según sus características, expresadas en TMR obtenida por Calorimetría Indirecta $(\mathrm{Cl})$, actividad física y estado nutricional, con una restricción de $500 \mathrm{kcal} /$ día respecto del gasto energético total (GET), ya que esta reducción energética favorece la pérdida de peso de entre 0,5 a $1 \mathrm{~kg}$ de peso corporal semanal ${ }^{19}$. Se elaboró una pauta alimentaria ajustada a los requerimientos de energía según sustratos expresados en el Valor Energético Total (VET).

Para el grupo "3 porciones de lácteos", la pauta alimentaria estuvo ajustada a requerimientos energéticos y nutricionales individuales, basada en porciones de alimentos con precisión de los sustratos energéticos en términos del $15-20 \%$ de las calorías provenientes de las proteínas ( $1 \mathrm{~g} /$ proteína/kg peso ideal), 50$60 \%$ de las calorías provenientes de los carbohidratos y $25 \%$ de las calorías provenientes de los lípidos. Los tiempos de comida y la distribución porcentual de energía que se indicaron fueron desayuno $25 \%$, colación de media mañana $7,5 \%$, almuerzo $35 \%$, colación de media tarde $7,5 \%$ y once $25 \%$, y se indicó el consumo de tres porciones de lácteos diarios $(720 \mathrm{mg}$ de calcio/día) como se menciona en las guías alimentarias para la población chilena ${ }^{3}$.

Para el grupo "5 porciones de lácteos", se consideraron las mismas características en la pauta alimentaria que para el grupo 1, pero ésta se ajustó con cinco porciones diarias de leche fluida UHT descremada, equivalente al aporte de 1.200 mg de calcio/día, por lo que se proporcionaron siete litros de forma semanal.

La verificación del grado de cumplimiento de la prescripción dietética, se realizó a través de la comparación de la pauta alimentaria entregada en la consulta nutricional de ingreso con el Registro Dietético Diario de siete días. Se compararon números, horarios, estructura, tiempos de comidas y porciones de lácteos.

A ambos grupos se les solicitó que mantuvieran la actividad física que realizaban, sin disminuir ni aumentar su frecuencia e intensidad. En los casos que no la realizaran, se les solicitó que mantuvieran esta condición durante el transcurso del estudio.

\section{Mediciones}

Se evaluó el estado nutricional por el método antropométrico, donde se determinó talla $(\mathrm{m})$ y peso $(\mathrm{kg})$, las cuales fueron utilizadas para el cálculo del índice de masa corporal, IMC $\left(\mathrm{kg} / \mathrm{m}^{2}\right)$, cuya clasificación se realizó según criterio $\mathrm{OMS}^{20}$. Además, se midió circunferencia de cintura (CC) expresada en $\mathrm{cm}$, realizada con la técnica estandarizada por la $\mathrm{OMS}^{21}$. Por otra parte, se obtuvo masa grasa $(\mathrm{kg})$ mediante la técnica de impedanciometría, y TMR mediante el método de $\mathrm{Cl}$. Todos los análisis fueron realizados por una nutricionista entrenada, quien utilizó protocolos estandarizados para cada una de las evaluaciones.

Se citó a las participantes, mediante una invitación enviada por correo electrónico, a una reunión grupal donde fueron instruidas sobre los requisitos necesarios para llevar a cabo la medición de la $\mathrm{Cl}$ y la bioimpedanciometría, para lo cual se les solicitó que el día previo al examen debían ayunar por 12 horas, reposar físicamente, haber orinado previamente, no haber consumido cafeína, alcohol y nicotina, no haber realizado ejercicio intenso, no presentar fiebre, no poseer enfermedad respiratoria que comprometa el intercambio gaseoso o eliminación de $\mathrm{CO}_{2}$ y frecuencia respiratoria normal. Además, se les solicitó que, para dirigirse al lugar de la medición, Centro de Vida Saludable (CVS) de la Universidad de Concepción, lo realizaran con el mínimo de esfuerzo físico posible para no alterar la medición. Una vez en el CVS las participantes debieron reposar por 15-20 minutos, y al momento de la medición, debieron mantener posición supina, sin dormir, tranquila y con respiración normal.

\section{Equipos}

Para la medición de talla se utilizó un estadiómetro marca SECA, modelo 213, con una precisión de $1 \mathrm{~mm}$, y para el peso, una balanza digital, marca TANITA, modelo TBF-300-A, con una precisión de 0,1 kg para peso. Además, se evaluó circunferencia de cintura $(\mathrm{cm})$, con una cinta métrica marca SECA, modelo 201, con una precisión de 0,1 mm. Para medir composición corporal, se utilizó el método de análisis de impedancia bioeléctrica, por lo cual se contó con una balanza con bioimpedanciómetro incorporado, marca TANITA, modeloTBF-300-A, con una precisión de $0,1 \%$ para masa grasa. Los niveles de masa grasa corporal expresados en kg y en porcentaje fueron obtenidos mediante esta medición.

La TMR al inicio y al término de la intervención dietoterapéutica, se determinó utilizando el método de $\mathrm{Cl}$ con el equipo Medgraphics, modelo ULTIMA CCM. Protocolos estandarizados fueron utilizados para realizar esta medición incluyendo la calibración del equipo previo a la medición de los participantes.

\section{Aspectos éticos}

Este estudio fue aprobado por el Comité de Ética, Bioética y Bioseguridad de la Vicerrectoría de Investigación y Desarrollo de la de la Universidad de Concepción y por el Comité Ético Científico del Servicio de Salud de Concepción. Todos los participantes fueron informados del objetivo del estudio y los protocolos de evaluación y registro de alimentos, otorgando la oportunidad de realizar consultas en relación a su participación. Una vez informados del estudio todos los participantes firmaron un consentimiento informado para tomar parte de este estudio.

\section{Análisis estadístico}

Debido al tamaño de la muestra y distribución de las variables se realizaron análisis con pruebas no paramétricas. 
Las características de los participantes según grupos de intervención Pre-test fueron presentadas como promedio y desviación estándar para variables continuas y como porcentaje para variables categóricas. Diferencias significativas entre los grupos de intervención fueron determinadas con la prueba Mann-Whitney para variables continuas y con la prueba de Fisher's para variables categóricas.

Para establecer si los cambios observados Post-test fueron significativos para cada uno de los grupos de intervención la prueba de Mann-Whitney fue aplicada. Para determinar si los cambios observados entre los grupos de intervención después de las 8 semanas de intervención fueron significativamente diferentes, se estimó el delta o cambio en variable de interés post intervención [Post Pre test] y sus respectivos $95 \%$ intervalos de confianza. De manera que un delta negativo indicó que hubo una reducción en la variable de interés, mientras que un delta positivo indicó que hubo un aumento en la variable. Los deltas de cada uno de los grupos fueron posteriormente comparados con la prueba de Mann-Whitney. Diferencias estadísticamente significativas fueron aceptadas con un valor $\mathrm{p}<0.05$. Todos los resultados fueron estimados con STATA.

\section{RESULTADOS}

La intervención se inició con 19 participantes (grupo 1 "3 porciones de lácteos" $\mathrm{n}=10$ y grupo 2 "5 porciones de lácteos" $n=9$ ), pero hubo un abandono durante la cuarta semana de la intervención, por lo que el estudio finalizó con 18 participantes (grupo 1 " 3 porciones de lácteos" $n=10$ y grupo 2 " 5 porciones de lácteos" $n=8$ ). Las características generales, antropométricas y de alimentación de ambos grupos se describen en la Tabla 1. En resumen, no se encontraron diferencias significativas para estas variables entre ambos grupos previos a la intervención, a excepción del consumo de carbohidratos y lípidos, el cual fue significativamente diferente entre los grupos de intervención.

En la Tabla 2, se presentan las variables antropométricas y de composición corporal, pre y post- intervención. Al término de las 8 semanas de intervención el grupo que restringió su consumo a 3 porciones de lácteos al día como el que consumió 5 porciones al día, redujeron significativamente sus niveles de peso corporal (-3,6 vs. $-3,4 \mathrm{~kg})$, IMC $\left(-1,45 \mathrm{vs.}-1,31 \mathrm{~kg} / \mathrm{m}^{2}\right)$, CC $(-4,5$ vs. $-6,2 \mathrm{~cm})$ y \%MG (-2,5 vs. $-2,7 \%$ ) (Figura 1 y Tabla 2$)$. A pesar de que ambos grupos redujeron significativamente los niveles de adiposidad, las reducciones logradas por ambos grupos intervenidos no fueron significativamente diferente entre sí (Tabla 2).

Tabla 1. Características generales de los grupos intervenidos.

\begin{tabular}{|c|c|c|c|}
\hline & $<3$ porciones & 5 porciones & Valor $\mathbf{P}$ \\
\hline Número de participantes & 10 & 8 & -- \\
\hline Edad (años) & 21,8 & 20,8 & 0.963 \\
\hline \multicolumn{4}{|l|}{ Características antropométricas } \\
\hline Peso $(k g)$ & $68,1 \pm 6,0$ & $69,9 \pm 7,3$ & 0.374 \\
\hline Talla $(\mathrm{m})$ & $1.58 \pm 0.04$ & $1.59 \pm 0.06$ & 0.688 \\
\hline $\mathrm{IMC}\left(\mathrm{kg} / \mathrm{m}^{2}\right)$ & $27,0 \pm 1,8$ & $27,2 \pm 1,5$ & 0.656 \\
\hline Circunferencia cintura & $86,2 \pm 5,9$ & $85,5 \pm 3,5$ & 0.688 \\
\hline$\%$ Masa grasa & $33,8 \pm 4,6$ & $35,3 \pm 2,9$ & 0.722 \\
\hline \multicolumn{4}{|l|}{ Alimentación } \\
\hline Ingesta energética (Kcal / día) & $1767,5 \pm 366,7$ & $1698,9 \pm 225,1$ & 0.594 \\
\hline Proteínas (\% VET / día) & $14,1 \pm 2,0$ & $14,5 \pm 2,5$ & 0.929 \\
\hline Carbohidratos (\% VET / día) & $57,7 \pm 5,3$ & $50,2 \pm 5,4$ & 0.012 \\
\hline Lípidos (\% VET / día) & $27,1 \pm 5,9$ & $33,7 \pm 4,9$ & 0.026 \\
\hline Calcio dietario (mg / día) & $483,8 \pm 213,9$ & $447,1 \pm 123,6$ & 0.859 \\
\hline Porciones de lácteos (numero/día) & $1,4 \pm 1,0$ & $1,9 \pm 0,8$ & 0.397 \\
\hline \multicolumn{4}{|l|}{ Niveles de actividad física (\%) } \\
\hline Leve & 80 & 87,5 & 0.247 \\
\hline Moderada & 20 & 12,5 & \\
\hline Intensa & 0 & 0 & \\
\hline
\end{tabular}

Datos presentados como promedio y desviación estándar para variables continuas y como porcentajes para variables categóricas. Las diferencias significativas entre variables continuas fueron determinadas con la prueba de Mann-Whitney y con la prueba de Fisher's exact test para variables categóricas. 

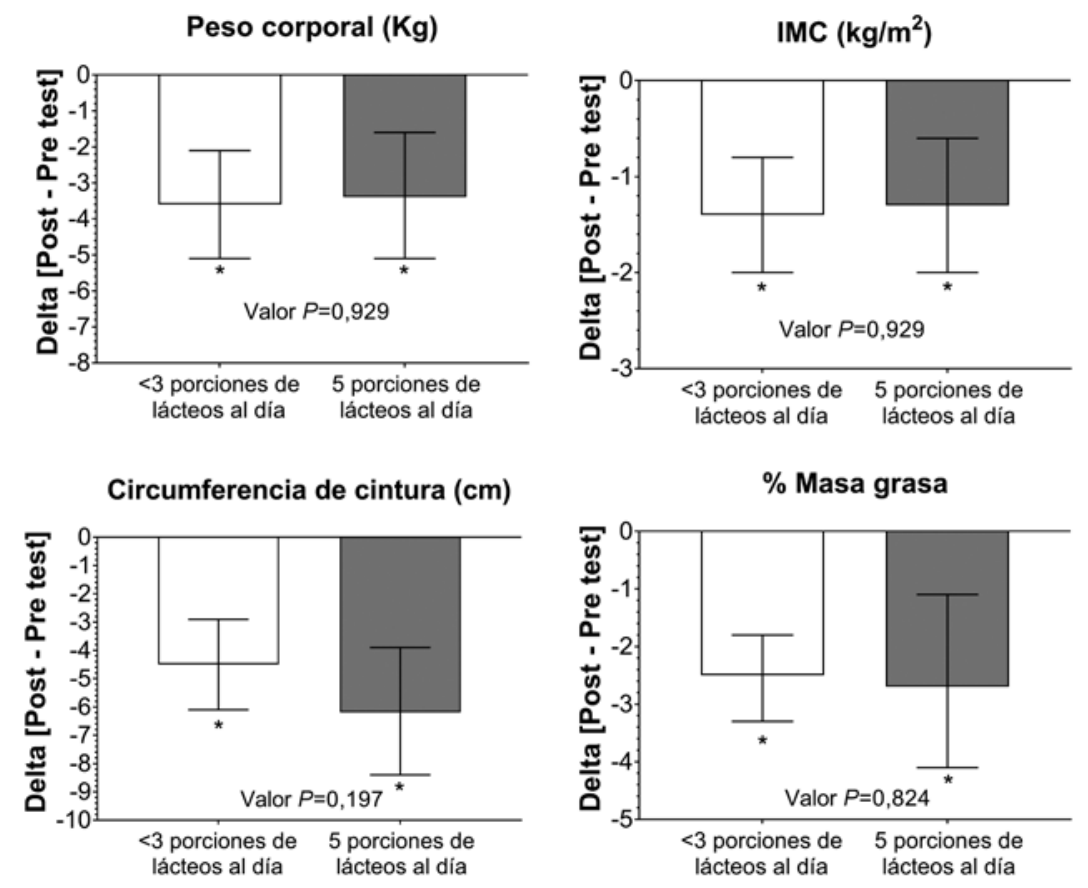

Figura 1. Cambios en variables antropométricas según grupo post intervención. Datos presentados como delta y 95\% IC para variables antropométricas posterior a 8 semanas de intervención. Diferencias en variables antropométricas entre Pre y Post intervención para cada uno de los (3 vs 5 porciones de lácteos diarios) fueron determinadas con la prueba de Mann-Whitney. El delta para cada uno de los grupos fue estimado como [Post test - Pre test]. El valor P indica si los cambios observados entre los grupos de intervención al término de esta fueron significativamente diferentes. El símbolo * indica si los cambios observados entre pre y post test para cada uno de los grupos fueron estadísticamente significativos. Diferencias estadísticamente significativas fueron aceptadas con un valor $\mathrm{p}<0,05$.

Tabla 2. Cambios en características antropométricas pre y post-intervención según grupo de intervención.

\begin{tabular}{|c|c|c|c|c|c|c|}
\hline \multirow[t]{2}{*}{ Variable } & \multirow[t]{2}{*}{ Grupo } & \multirow[t]{2}{*}{ Pre test } & \multirow[t]{2}{*}{ Post test } & \multicolumn{2}{|c|}{ Delta $(\Delta)[$ Post - Pre $]$} & \multirow{2}{*}{$\begin{array}{l}\text { Valor } \mathbf{P} \text { para } \Delta \\
\text { entre grupos* }\end{array}$} \\
\hline & & & & $\Delta(95 \%$ IC $)$ & Valor $\mathbf{P}$ & \\
\hline \multirow[t]{2}{*}{ Peso (kg) } & $<3$ porciones & $68,1 \pm 6,0$ & $64,4 \pm 6,2$ & $-3,6(-5,1 ;-2,1)$ & 0,007 & 0,929 \\
\hline & 5 porciones & $69,9 \pm 7,3$ & $66,6 \pm 8,4$ & $-3,4(-5,1 ;-1,6)$ & 0,020 & \\
\hline \multirow[t]{2}{*}{ IMC (kg/m2) } & $<3$ porciones & $27,0 \pm 1,8$ & $25,5 \pm 1,8$ & $-1,4(-2,0 ;-0,8)$ & 0,006 & 0,920 \\
\hline & 5 porciones & $27,2 \pm 1,5$ & $25,9 \pm 2,2$ & $-1,3(-2,0 ;-0,6)$ & 0,017 & \\
\hline \multirow[t]{2}{*}{$\mathrm{CC}(\mathrm{cm})$} & $<3$ porciones & $86,2 \pm 5,9$ & $81,6 \pm 6,2$ & $-4,5(-6,1 ;-2,9)$ & 0,005 & 0,197 \\
\hline & 5 porciones & $85,5 \pm 3,5$ & $79,3 \pm 4,1$ & $-6,2(-8,4 ;-3,9)$ & 0,011 & \\
\hline \multirow[t]{2}{*}{ MG (\%) } & $<3$ porciones & $33,8 \pm 4,6$ & $31,2 \pm 5,3$ & $-2,5(-3,3 ;-1,8)$ & 0,005 & 0,824 \\
\hline & 5 porciones & $35,3 \pm 2,9$ & $32,6 \pm 4,2$ & $-2,7(-4,1 ;-1,1)$ & 0,017 & \\
\hline
\end{tabular}

Datos presentados como promedio y desviación estándar para las medidas antropométricas tomadas Pre y Post intervención. Diferencias en variables antropométricas entre Pre y Post intervención para cada uno de los grupos ( 3 vs 5 porciones de lácteos diarios) fueron determinadas con la prueba de Mann-Whitney. El delta para cada uno de los grupos fue estimado como [Post test - Pre test] y los resultados presentados como delta promedio con su respectivo 95\% intervalos de confianza (95\% IC) para cada grupo de intervención. *Para evaluar si el delta alcanzado en el grupo de 3 y 5 porciones de lácteos al día fue significativamente diferente se ejecutó la prueba de Mann-Whitney. Diferencias estadísticamente significativas fueron aceptadas con un valor $\mathrm{p}<0,05$. Grupos de intervención corresponden a 3 porciones de lácteos diarios y aquellos que recibieron 5 porciones de lácteos diarios, IMC: índice de masa corporal; CC: circunferencia de cintura; MG: masa grasa. 
En la tabla 3, se muestran los cambios en la ingesta energética, de macronutrientes, calcio dietario y porciones de lácteos, pre y post intervención en ambos grupos intervenidos. Se puede observar que la ingesta energética post-intervención disminuyó significativamente para ambos grupos con un delta de $-552,2 \mathrm{kcal} /$ día para el grupo "3 porciones de lácteos" $(p=0.005)$ y $-268,4 \mathrm{kcal} /$ día para el grupo " 5 porciones de lácteos" ( $p=0.049)$. El consumo de proteínas aumentó significativamente en el grupo de 3 y 5 porciones $(1,8$ vs. $3,7 \% \mathrm{VET} /$ día), respectivamente. El consumo de lípidos disminuyó significativamente en el grupo de "5 porciones" pero no se observaron cambios significativos para en el grupo de " 3 porciones" (-11,3 vs $-2,6 \%$ VET/ día, respectivamente). Mientras que el consumo de carbohidratos aumentó significativamente en el grupo de 5 porciones, pero no en el de 3 porciones $(8,8$ vs $1,4 \%$ VET/día, respectivamente). Para el consumo de calcio sólo se observaron cambios significativos en el grupo de 5 porciones (916,4 mg/día) mientras que para el grupo de 3 porciones no se modificó significativamente (-28,9 mg/día) (Figura 2).

Tabla 3. Ingesta energética, de macronutrientes, calcio dietario y porciones de lácteos pre y post-intervención según grupo de intervención.

\begin{tabular}{|c|c|c|c|c|c|c|}
\hline \multirow[t]{2}{*}{ Variable } & \multirow[t]{2}{*}{ Grupo } & \multirow[t]{2}{*}{ Pre test } & \multirow[t]{2}{*}{ Post test } & \multicolumn{2}{|c|}{ Delta $(\Delta)[$ Post - Pre] } & \multirow{2}{*}{$\begin{array}{l}\text { Valor } \mathbf{P} \text { para } \Delta \\
\text { entre grupos* }\end{array}$} \\
\hline & & & & $\Delta(95 \%$ IC $)$ & Valor $\mathbf{P}$ & \\
\hline Ingesta energética & $<3$ porciones & $1767,5 \pm 366,7$ & $1215,3 \pm 147,4$ & $-552,2(-829,4 ;-274,9)$ & 0,005 & 0,130 \\
\hline (Kcal / día) & 5 porciones & $1698,9 \pm 225,1$ & $1430,5 \pm 135,0$ & $-268,4(-486,7 ;-49,9)$ & 0,049 & \\
\hline Proteínas & $<3$ porciones & $14,1 \pm 2,0$ & $15,8 \pm 1,5$ & $1,8(0,4 ; 3,0)$ & 0,016 & 0,155 \\
\hline (\% VET/día) & 5 porciones & $14,5 \pm 2,5$ & $18,2 \pm 1,0$ & $3,7(1,3 ; 6,0)$ & 0,025 & \\
\hline Carbohidratos & $<3$ porciones & $57,7 \pm 5,3$ & $59,1 \pm 5,8$ & $1,4(-2,5 ; 5,4)$ & 0,202 & 0,026 \\
\hline (\% VET/día) & 5 porciones & $50,2 \pm 5,4$ & $58,7 \pm 5,5$ & $8,5(3,2 ; 13,9)$ & 0,017 & \\
\hline Lípidos & $<3$ porciones & $27,1 \pm 5,9$ & $24,5 \pm 3,6$ & $-2,6(-6.4 ; 1.2)$ & 0,092 & 0,010 \\
\hline (\% VET/día) & 5 porciones & $33,7 \pm 4,9$ & $22,4 \pm 4,6$ & $-11,3(-16,7 ;-5,9)$ & 0,011 & \\
\hline Calcio dietario & $<3$ porciones & $483,8 \pm 213,9$ & $454,9 \pm 211,3$ & $-28,9(-190,5 ; 132,5)$ & 0,386 & 0,0004 \\
\hline Total (mg/día) & 5 porciones & $447,1 \pm 123,6$ & $1363,6 \pm 88,4$ & $916,4(802,5 ; 1030,3)$ & 0,011 & \\
\hline \multirow[t]{2}{*}{$\begin{array}{l}\text { Porciones lácteos } \\
\text { (número/día) }\end{array}$} & $<3$ porciones & $1,4 \pm 1,0$ & $1,8 \pm 0,6$ & $0,4(0,2 ; 1,0)$ & 0,165 & 0,0001 \\
\hline & 5 porciones & $1,9 \pm 0,8$ & $5,0 \pm 0,0$ & $3,1(2,4 ; 3,8)$ & 0,010 & \\
\hline
\end{tabular}

Datos presentados como promedio y desviación estándar para ingesta energética, macronutrientes, calcio y porciones de lácteos Pre y Post intervención. Diferencias en variables antropométricas entre Pre y Post intervención para cada uno de los grupos ( 3 vs 5 porciones de lácteos diarios) fueron determinadas con el promedio y su respectivo 95\% intervalos de confianza (95\% IC) para cada grupo de intervención. *Para evaluar si el delta alcanzado en el grupo de 3 y 5 porciones de lácteos al día fue significativamente diferente se ejecutó la prueba de Mann-Whitney. Diferencias estadísticamente significativas fueron aceptadas con un valor $\mathrm{p}<0,05$. Grupos de intervención corresponden a 3 porciones de lácteos diarios y aquellos que recibieron 5 porciones de lácteos diarios, VET: Valor energético total. prueba de Mann-Whitney. El delta para cada uno de los grupos fue estimado como [Post test - Pre test] y los resultados presentados como delta. 
Ingesta energética (Kcal)

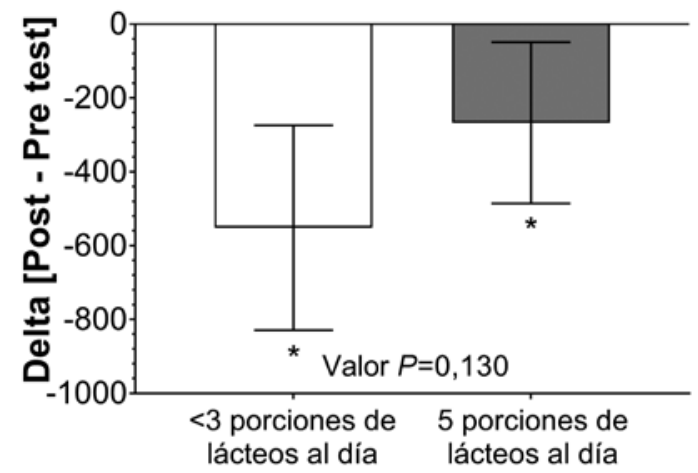

Carbohidratos (\% VET / día)

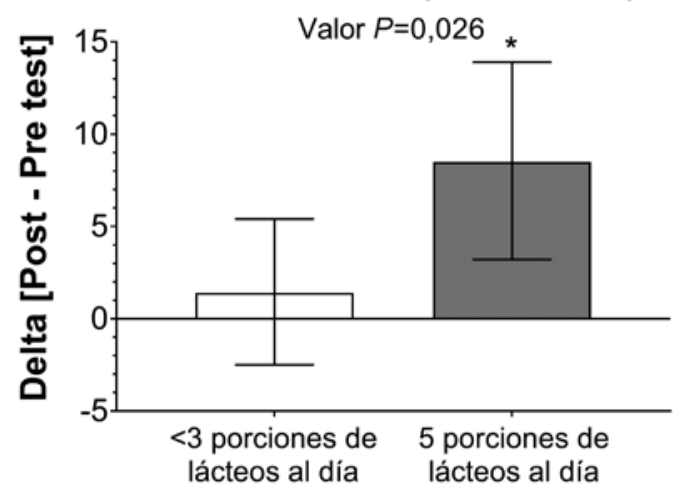

Calcio dietario ( $\mathrm{mg} /$ día)

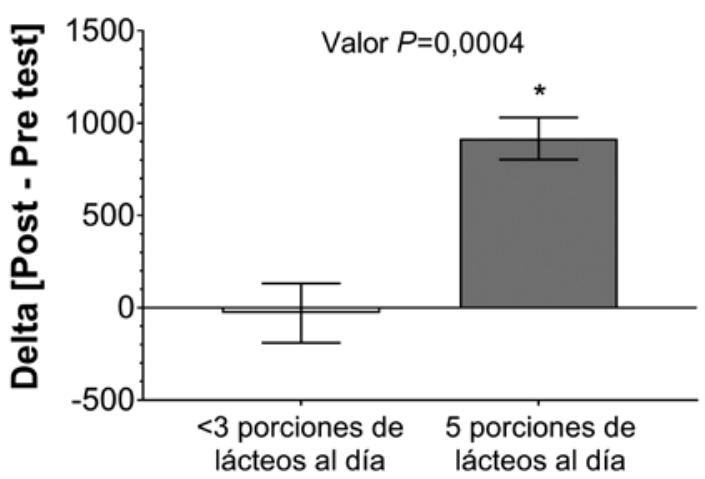

Proteínas (\% VET / día)

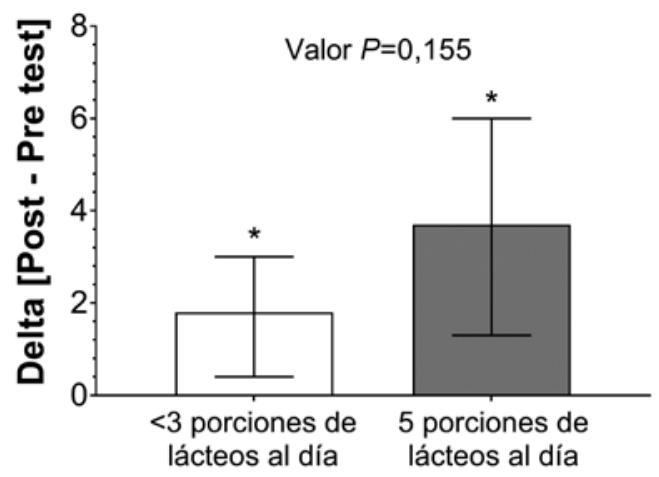

Lípidos (\% VET / día)

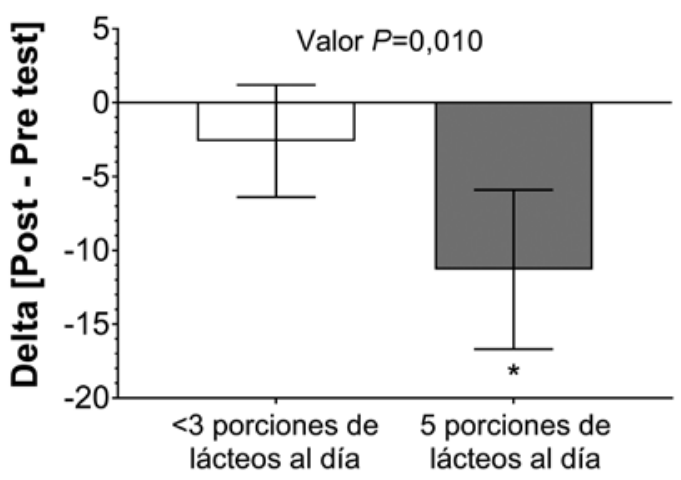

Porciones de lácteos al día

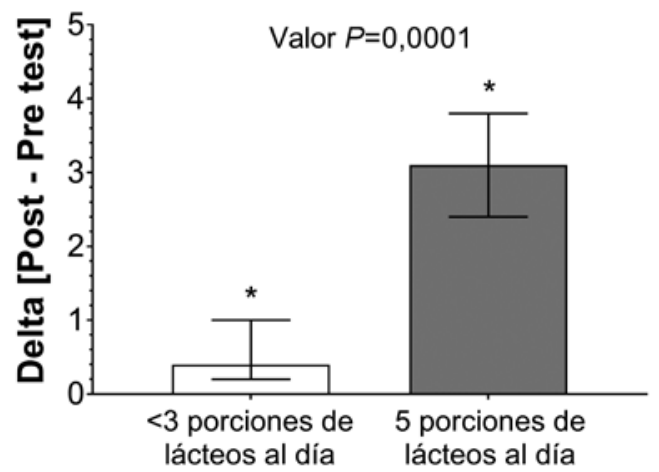

Figura 2. Cambios en la ingesta energética, macronutrientes, consumo de calcio dietario y porciones de lácteos según grupo de intervención. Datos presentados como delta y 95\% IC para variables de alimentación posterior a 8 semanas de intervención. Diferencias en estas variables entre Pre y Post intervención para cada uno de los grupos (3 vs 5 porciones de lácteos diarios) fueron determinadas con la prueba de Mann-Whitney. El delta para cada uno de los grupos fue estimado como [Post test - Pre test]. El valor P indica si los cambios observados entre los grupos de intervención al término de esta fueron significativamente diferentes. El símbolo * indica si los cambios observados entre pre y post test para cada uno de los grupos fueron estadísticamente significativos. Diferencias estadísticamente significativas fueron aceptadas con un valor $\mathrm{p}<0,05$. 


\section{DISCUSIÓN}

La etapa universitaria tiene una directa trascendencia sobre los hábitos alimentarios en la población joven. Para mejorar los hábitos alimentarios, la evidencia describe que las intervenciones dietoterapéuticas, deben basar sus indicaciones en alimentos y en grupos de alimentos, con las cuales se pueden obtener cambios en las medidas antropométricas de los pacientes ${ }^{3,22}$. Los resultados obtenidos en este estudio en relación al peso corporal, IMC, MG\% y CC, evidenciaron que en ambos grupos se produjeron disminuciones significativas, que pudieran deberse a la restricción energética de $500 \mathrm{kcal} / \mathrm{d}$ ía en la prescripción dietética según su TMR determinada por $\mathrm{Cl}$. Resultados similares fueron presentados por Arrebolas y colaboradores en España, en el cual se realizó una intervención dietoterapéutica por 6 meses con restricción energética de $500 \mathrm{kcal} / \mathrm{d}$ áa respecto de su ingesta diaria estimada y consiguió una pérdida significativa del peso corporal, IMC, CC y MG $\%{ }^{24}$.

La evidencia señala que frente a una intervención dietoterapéutica con restricción energética, la pérdida de peso alcanzada, manifiesta dentro de los primeros cambios antropométricos, la disminución de la $\mathrm{CC}^{25,26}$. En este estudio los resultados obtenidos, establecen diferencias, aunque no significativas, entre el grupo "3 porciones de lácteos" y "5 porciones de lácteos" en relación a la CC, siendo el grupo " 5 porciones de lácteos" quien evidenció una mayor reducción $(-6,2 \mathrm{~cm})$ en comparación con el grupo "3 porciones de lácteos" $(-4,5 \mathrm{~cm})$. Si bien estas diferencias entre grupos fueron no significativas esto podría estar explicado por la corta duración del estudio y el tamaño reducido de la muestra. Ya que la revisión sistemática y meta-análisis realizado por realizada por Abargouei y colaboradores, donde reportó que el aumento en la ingesta de lácteos dio resultados de una mayor reducción en la CC respecto del grupo control, pero sólo cuando esta mayor ingesta estaba acompañada de una restricción calorica ${ }^{27}$. Otro estudio que corrobora el efecto de la ingesta de calcio sobre la reducción de parámetros de adiposidad es el realizado por Zemel y colaboradores en Estados Unidos, donde se intervino a dos grupos de obesos, a través de la restricción energética con diferencias en el aporte de ingesta de calcio durante 12 semanas, se obtuvieron cambios significativos en las mediciones de MG, MLG, peso corporal y CC en el grupo suplementado con calcio aportado por lácteos (1100 mg de calcio/día) respecto del grupo control ${ }^{23}$. El rol del calcio dietario ha obtenido gran interés en los últimos años, ya que se ha evidenciado que un aumento en la ingesta de este, incrementa la pérdida de grasa corporal favoreciendo su redistribución alejada del abdomen, lo que estaría relacionado con la disminución la lipogénesis y el aumento de la lipólisis, sugiriendo una asociación inversa entre el peso corporal, porcentaje de grasa corporal y la ingesta de calcio dietario ${ }^{14,28,29}$. Sin embargo, otros estudios refieren que cuando se aumenta el consumo de lácteos sin restricción energética, no se producen cambios en la composición corporal, pero si el aumento en el consumo de porciones de lácteos se realiza en conjunto con una dieta con restricción energética, produce una mayor pérdida de peso, de CC y reducción de la MG respecto de un grupo control ${ }^{11,12,29}$. Esto podría explicar los resultados de este estudio, donde se observaron reducciones significativas para ambos grupos en las variables del estado nutricional estudiadas, podría explicarse por la restricción calórica de 500 kcal más que al aumento en el consumo de calcio. Si bien se ha planteado que un aumento en la ingesta de calcio a través de la dieta (lácteos) con restricción energética pueda tener efectos sobre el peso corporal a corto plazo; estudios a largo plazo, han mostrado una disminución significativa en la prevalencia de obesidad, lo cual podría estar asociado a que personas que consumen suficiente calcio a través de su alimentación diaria posiblemente tendrían una alimentación más saludable que aquellas personas que no consumen productos lácteos ${ }^{11,12,15}$.

Si bien este estudio fue realizado con la aplicación de metodologías estandarizadas, es importante considerar los resultados de este estudio dentro del marco de sus limitaciones. Una de las limitaciones de este estudio tiene relación al pequeño tamaño muestral, sin embargo, a pesar de incluir a 18 personas en la intervención, se lograron observar cambios significativos en las variables del estado nutricional, lo que indica que el tamaño de la muestra fue suficiente para encontrar cambios significativos. Otro aspecto que es importante considerar es la duración el estudio la cual fue de corto plazo, y si bien produjo cambios significativos, sería importante verificar estos resultados en estudios de mediano (3-6 meses) y largo plazo (duración de al menos 12 meses), para poder comprobar los verdaderos resultados del aumento en la ingesta de calcio sobre las variables del estado nutricional. Estudios futuros podrían ser realizados en población masculina, pero también en población con niveles más severos de obesidad, para establecer si el consumo de calcio podría asociarse a cambios en los niveles de adiposidad de estos grupos. Por último, la no inclusión de un grupo de control en esta intervención no permite establecer si los cambios observados fueron debido al azar.

\section{CONCLUSIONES}

Estos resultados sugieren que los efectos de incrementar el consumo de calcio a través de la ingesta diaria de 5 porciones de productos lácteos, no presenta beneficios adicionales en la mejora de las variables del estado nutricional en comparación al grupo que consume $<3$ porciones de lácteos al día, después de una intervención de 8 semanas. Esto indica que los cambios observados en el estado nutricional son debidos a la restricción calórica más que el consumo elevado de calcio.

Agradecimientos. Al Centro de Vida Saludable de la Universidad de Concepción, por el apoyo entregado para poder realizar este estudio, y al programa de Magíster en Nutrición Humana de la Universidad de Concepción por el financiamiento otorgado. 


\section{BIBLIOGRAFÍA}

1. MINSAL. National Health Survey 2016- 2017. Ministerio de Salud Chile. 2018.

2. Ratner R, Hernández P, Martel I \& Atalah E. Quality of nutrition and nutritional status in university students from 11 regions of Chile Rev Med Chile 2012; 140: 1571-1579.

3. MINSAL. Study to review and update the dietary guidelines for the Chilean population. Ministerio de Salud de Chile 2013.

4. Crovetto $M$, Figueroa B, González $L$, Jeria A \& Ramírez N. Dietary guidelines and their compliance in university students, Valparaíso, 2013, Chile. Rev Chil Nutr 2015; 42(2).

5. Cervera F, Serrano R, Vico C, Milla M \& García M. University eating habits and nutritional assessment in a university population. Nutr Hosp 2013; 28(2): 438-446.

6. Ledo-Varela M, De Luis Román D, González-Sagrado M, Izaola $O$, Conde $R$, \& Aller de la Fuente $R$. Nutritional characteristics and lifestyle in university students. Nutr Hosp 2011; 26(4): 814-818.

7. Hernández M. Dietoterapia. La Habana: Editorial Ciencias Médicas. 2008; cap. 8 p 62-65.

8. Manrique M, De la Maza M, Carrasco F, Moreno M, Albala C, García J. et Al. Diagnosis, evaluation and non-pharmacological treatment of overweight or obese patients. Rev Med Chile 2009; 137: 963-971.

9. Pino J, López $M$, Cofré $M$, González C \& Reyes L. Foodnutritional knowledge and nutritional status of fourth-year students according to private and subsidized establishments of the city of Talca. Rev Chil Nutr 2010; 37(4): 418-426.

10. MINSAL. Final Report National Food Consumption Survey. Ministerio de Salud Chile. 2015.

11. Soares $M$, Murhadi L, Kurpad A, Chan She Ping-Delfos $W$ \& Piers L. Mechanistic roles for calcium and vitamin $D$ in the regulation of body weight regulation of body weight. Obes Rev 2012; 13: 592-605.

12. Heaney R. Normalizing calcium intake: projected population effects for body weight. I Nutr 2003; 133: 268S-270S.

13. Onakpoya I, Perry R, Zhang I \& Ernst E. Efficacy of calcium supplementation for management of overweight and obesity: systematic review of randomized clinical trials. Nutr Rev 2011; 69: 335-343.

14. Zemel M, Shi H, Greer B, Dirienzo D \& Zemel P. Regulation of adiposity by dietary calcium. FASEB J 2000; 14: 1132-1138.

15. Soares $M \&$ Chan She-Ping-Delfos W. Postprandial energy metabolism in the regulation of body weight: is there a mechanistic role for dietary calcium? Nutrients 2010; 2: 586-590.

16. Teegarden D, White $K$, Lyle R, Zemel M, Van Loan $M$,
Matkovic V, et Al. Calcium and Dairy Product Modulation of Lipid Utilization and Energy Expenditure. Obesity. 2008; 16: 1566-1572.

17. EFSA. Scientific Opinion on the Tolerable Upper Intake Level of calcium. EFSA Journal 2012; 10(7): 2814.

18. Cervera F, Serrano R, Vico C, Milla M \& García M. Quality of the diet in university students with different academic profiles. Nutr Hosp 2015; 31(5): 2230-2239.

19. SEEDO. Prevention, diagnosis and treatment of obesity. Sociedad Española para el Estudio de la Obesidad. 2016

20. WHO. Body mass index. World Health Organization.

21. WHO. Waist circumference and waist-hip ratio: Report of a WHO expert consultation, Geneva. World Health Organization. 2008.

22. Ball $L$, Leveritt $M$, Cass $S$ \& Chaboyer W. Effect of nutrition care provided by primary health professionals on adults' dietary behaviours: a systematic review. Family Practice, Published by Oxford University Press 2015; 32(6): 605-617.

23. Zemel MB, Richards J, Mathis S, Milstead A, Gebhardt L \& Silva E. Dairy augmentation of total and central fat loss in obese subjects. International Journal of Obesity 2005; 29: 391-397.

24. Arrebola E, Gómez-Candela C, Fernández C, Bermejo L \& Loria V. Efficacy of a program for the treatment of overweight and non-morbid obesity in primary care and its influence on the modification of lifestyles. Nutr Hosp 2013; 28(1): 137-141.

25. Bulló M, Garcia-Aloy M, Martínez-González MA, Corella D, Fernández-Ballart JD, Fiol $M$ et Al. Association between a healthy lifestyle and general obesity and abdominal obesity in an elderly population at high cardiovascular risk. Prev Med 2011; 53(3): 155-161.

26. Tárraga M, Rosich N, Panisello J, Gálvez A, Serrano J, RodríguezMontes J \& Tárraga P. Efficacy of motivation strategies in the treatment of overweight and obesity. Nutr Hosp 2014; 30(4): 741-748.

27. Abargouei AS, Janghorbani $M$, Salehi-Marzijarani $M$ \& Esmaillzadeh A. Effect of dairy consumption on weight and body composition in adults: a systematic review and metaanalysis of randomized controlled clinical trials. International Journal of Obesity 2012; 36(12): 1485-1493.

28. Aguilera $R$, Jorquera $P$, Salgado $C \&$ Flores $C$. Calcium supplementation for reducing weight in people with obesity; an overview of systematic reviews. Nutr Hosp 2016; 33(2): 464-471.

29. Van Loan M. The role of dairy foods and dietary calcium in weight management. I Am Coll Nutr 2009; 28(Suppl 1): 120S-129S. 